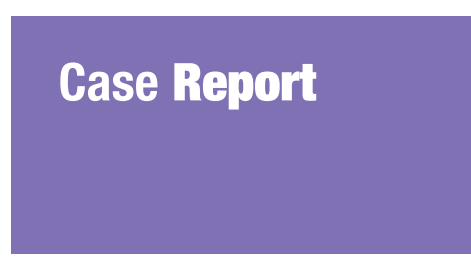

Submitted: 17 Apr 2015

Accepted: 12 Dec 2016

Online: 30 Jun 2017

\section{Compound Odontoma in Anterior Mandible-A Case Report}

\author{
Eswara UMA
}

Department of Pediatric Dentistry, Faculty of Dentistry, Melaka Manipal Medical College, Melaka, Malaysia

To cite this article: Uma E. Compound odontoma in anterior mandible-a case report. Malays $J$ Med Sci. 2017;24(3):92-95. https://doi.org/10.21315/mjms2017.24.3.11

To link to this article: https://doi.org/10.21315/mjms2017.24.3.11

\begin{abstract}
Odontomas are the most common odontogenic hamartomas worldwide. Depending on the level of organisation of the tissues inside, these can be differentiated into compound type or complex type. As these are asymptomatic and do not cause any changes in the bone, they are often diagnosed during the routine dental examination. Complex odontomas are commonly found to occur in posterior mandible while compound odontomas are found in the anterior maxilla. A nine-year-old female child reported for a routine dental check-up, when a missing left permanent mandibular lateral incisor [32] was noticed. Further investigations revealed compound odontoma and unerupted 32, which is an unusual location. Early detection of these tumours is essential to avoid lengthy corrective treatments.
\end{abstract}

Keywords: odontoma, compound odontoma, odontogenic tumor, impacted tooth

\section{Introduction}

Odontomas were first described by Paul Brocain in 1867. He used the term odontoma for all odontogenic tumours; however, currently, the usage of the term has become much more restricted (1). Odontomas are now considered as hamartomatous odontogenic lesions as they comprise of both epithelial and ectomesenchymal components, having morphologically normal cells with defective structural organisation (2). Odontomas are included in the WHO classification of head and neck tumours as a group of lesions affecting the odontogenic epithelium with odontogenic ectomesenchyme, with or without hard tissue formation (3). These hamartomas have been described as either complex type or compound type. In complex odontoma, the enamel, dentin, and cementum are present in a disorganised manner, whereas in compound odontoma, varied numbers of tooth-like elements are present (3).

Odontomas are characterised by slow and painless growth and maybe associated with retention of primary tooth or delay in the eruption of primary and permanent teeth (4).

Several factors have been associated with the pathogenesis of odontomas. These are trauma in the primary dentition period, hereditary anomalies like Gardner's syndrome, hyperactivity of odontoblasts or changes in the genetic components responsible for dental development (5).

While posterior mandible and anterior maxilla have been reported to be the most common location for complex odontoma, compound odontomas are mostly found in the anterior maxilla (6). This case report presents an unusual case of compound odontoma in the anterior mandible of a nine-year-old child.

\section{Case Report}

A healthy nine-year-old girl reported to the dental clinic for a routine dental check-up. Intra-oral examination revealed mixed dentition with no decayed teeth. It was found that her 
deciduous mandibular left lateral incisor [72] was present, whereas her permanent mandibular left lateral incisor [32] was missing. Initially, a provisional diagnosis of missing 32 was done.

Intra-oral radiograph revealed multiple radio-opaque tooth-like structures close to the root apex of 72 and the presence of 32 (Figure 1). This led to the diagnosis of compound odontoma. As the child and her parents were apprehensive of undergoing any surgical procedure on the dental chair, surgical enucleation of the odontoma was planned under general anaesthesia.

Pre-operative investigations included routine blood tests and pre-anaesthetic evaluation. Under general anaesthesia, perioral

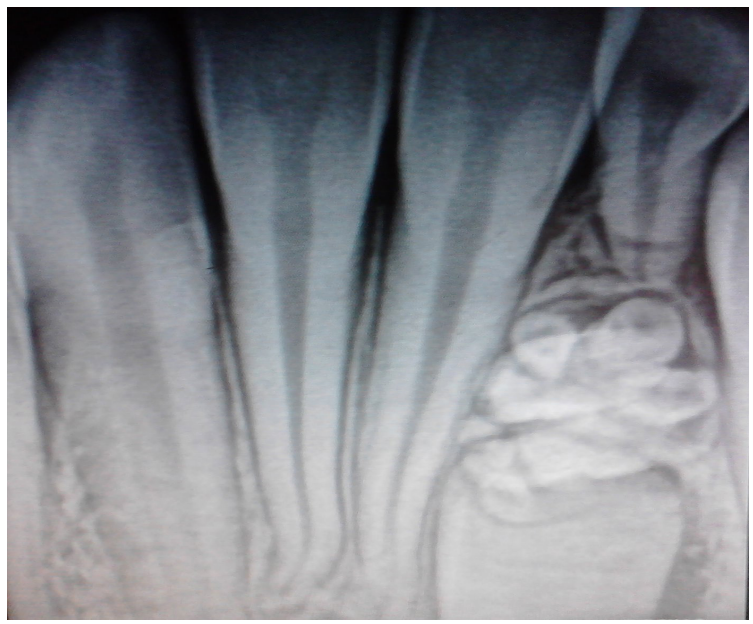

Figure 1. IOPA of 72 showing odontoma and 32

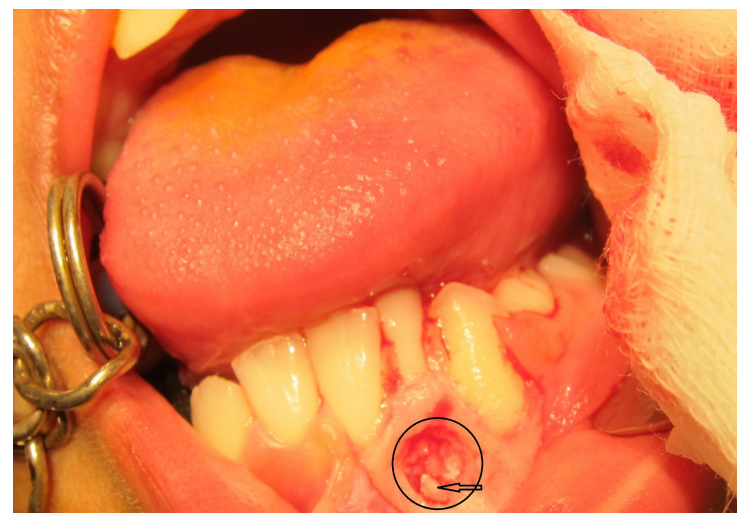

Figure 2. Surgical site showing denticles structures were prepared with betadine, and a rectangular mucoperiosteal flap was raised in the regions of 31,32 and 73 to expose the bone (Figure 2). A window was made in the bone close to the apex of 72 using a straight slow-speed handpiece bearing a round tungsten carbide bur under normal saline irrigation. Denticles were exposed, and a total of nine denticles of various sizes and shapes were removed along with the follicle (Figure 3). After the extraction of 72 , the position of 32 was determined. After thorough irrigation of the enucleated site, the flap was repositioned and sutured with 3-0 Vicryl. Histopathological examination of the denticles was done, and compound odontoma was diagnosed.

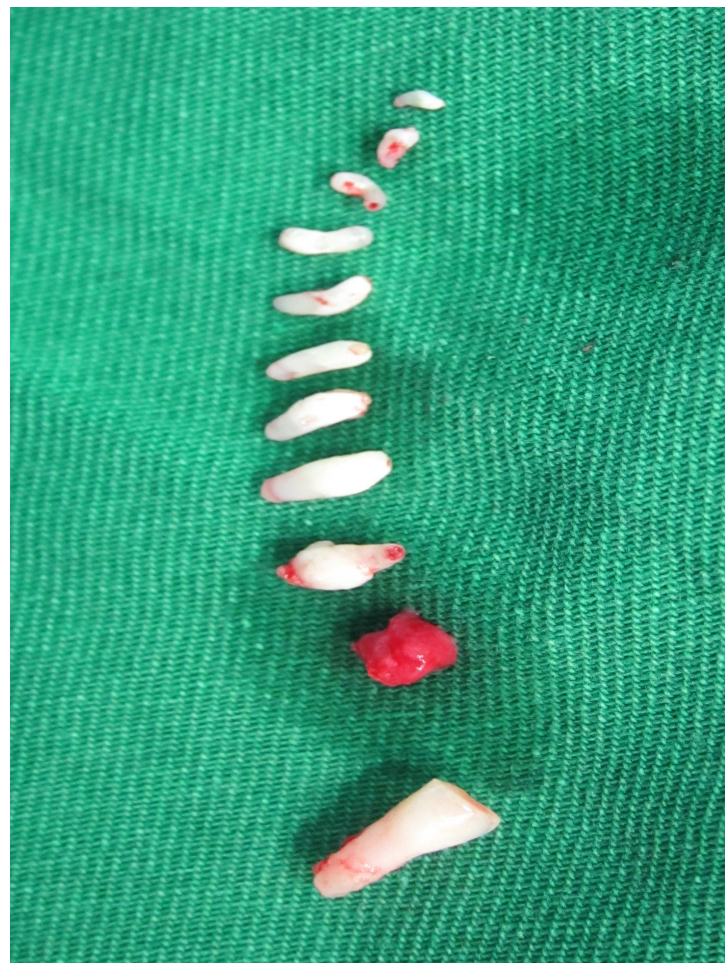

Figure 3. Enucleated nine denticles with follicle and extracted deciduous tooth 


\section{Discussion}

In the literature, the incidence of odontomas has been reported to range from $20 \%$ to $67 \%$ of all odontogenic neoplasms. While odontomas are pseudo-tumours made up of both epithelial and mesenchymal cells, they rarely lead to the pathological development of cysts, such as a calcifying odontogenic cyst or a dentigerous cyst (4).

Odontomas show several variations with regard to the age of occurrence, gender predilection and the number of denticles removed. Several studies have documented the age of occurrence and gender predilection of odontomas $(2,4)$. It has been stated that although odontomas may be diagnosed at any age, it is usually detected before the age of 20 years $(7,8)$. Complex odontoma has been known to have a slight predilection towards females, whereas compound odontoma is more common in males, contrary to previous reports that claimed there was no gender predilection $(2,4)$. The number of denticles removed during enucleation of odontomas ranged from 4 to 37 ; however, a recent case reported the removal of 232 denticles of a patient $(9,10,11)$.

Since a lot of variation is seen with regard to the age of occurrence of odontoma, it has been said that the age and location predilection for these malformations are on account of them being pathogenetically different. It has been postulated that since complex odontomas are considered to be a terminal stage lesion that is a hamartoma, they are seen in older people at the time of diagnosis with a predisposition for posterior mandibular regions. However, local hyperactivity of the dental lamina has been found to be the reason for the formation of compound odontomas (2). The present case corroborates with the hypothesis that compound odontoma is generally seen in younger people, as hyperactivity of the dental lamina is found to be high among them. However, in this case, the trigger for hyperactivity of the dental lamina is not known. There was no familial history nor history of trauma or infection as speculated in earlier studies.

In most cases, it is found to be associated with an impacted tooth (12). Unless they are superficial odontomas that rarely cause expansion of bone, odontomas are diagnosed on the basis of radiographs taken after primary complaint of the patient. In our case, odontoma was diagnosed during a routine dental check-up when a retained deciduous tooth was observed with its successor missing. An intra-oral radiograph taken to rule out missing permanent lateral incisor revealed several tooth-like structures underneath the root of the deciduous tooth and above the crown of the unerupted permanent lateral incisor. Odontomas are known to cause eruption disturbances in the form of delayed eruption, impaction of teeth, over retention of primary teeth or abnormalities in the alignment of teeth-like tipping or displacement of adjacent teeth.

Studies have shown that compound odontomas are seen more frequently in the anterior maxilla (1). In the present case, odontoma was found in the anterior mandible, which is an uncommon site of occurrence. A previous report had mentioned a greater frequency for the odontomas to arise in the region of incisors and canines (9), which is confirmed in our case. However, the tendency of the odontomas to occur on the right side (1) of the jaw was contrary to our finding.

Surgical exposure followed by enucleation of the odontoma is the accepted choice of treatment in order to allow the eruption of the permanent tooth. Small-sized odontomas do not pose any difficulty while removal; however, the proximity to nearby structures must be kept in mind to prevent unnecessary injury to them. It is further recommended that in the case of an impacted tooth associated with odontoma, it is better to wait for three months for the eruption of the impacted tooth. In case the impacted tooth fails to erupt after three months, it is recommended that the impacted tooth should be surgically exposed with or without orthodontic traction (3).

In the present case, the retained 72 was extracted, and the odontoma was enucleated to allow the eruption of the permanent tooth. Generally, this procedure is done under local anaesthesia, but in the present case, due to apprehensions of the child and her parents, the procedure was done under general anaesthesia as a day care procedure. The specimen enucleated was sent for histopathology to rule out ameloblastic fibro odontomas and odonto ameloblastomas since these have a great resemblance to common odontomas, especially in the radiographic examination (8). A follow-up was recommended to plan for the management of the unerupted 32 . 


\section{Conclusion}

The present case report is of a compound odontoma in anterior mandible, associated with a retained primary tooth. The high possibility of a retained deciduous tooth and its unerupted successor associated with odontoma is highlighted. This was diagnosed in a routine dental check-up for a healthy child with cariesfree dentition. In order to prevent adverse effects of odontomas, the author suggests that greater emphasis should be given on routine dental check-ups for children so that these anomalies can be detected earlier, thereby, minimising the interventions needed after enucleation.

\section{Correspondence}

Dr Eswara Uma

MDS (Post Graduate institute of Medical Education and Research, Chandigarh, India)

Professor and Head of Department

Department of Pedodontics, Faculty of Dentistry

Melaka Manipal Medical College,

Jalan Batu Hampar, Bukit Baru,

75150 Melaka, Malaysia.

Tel: 6062896662 ext 3820

Fax: 6062817977

E-mail: eswara.uma@manipal.edu.my

\section{References}

1. Helena S, Pedro M. Compound odontomacase report. Rev Port Estomatol Med Dent Cir Maxilofac. 2013;54(3):161-165.

2. Patricia GA, Harumi I, Yuji M, et al. Podoplanin expression in odontomas: clinicopathological study and immunohistochemical analysis of 86 cases. J Oral Sci. 2011;53(1):67-75.

3. Prætorius F, Piattelli A. Odontogenic tumours. In: Barnes L, Evenson JW, Reichart PA, Sindransky D, editors. WHO classification of tumours. Pathology \& genetics. Head and neck tumours. Lyon, France: IARC Press; 2005. p 310-311.
4. Paolo B, Emanuele Z, Fabio R, Cesare G. Complex and compound odontomas. $J$ Craniofac Surg. 2012;23:685-688.

5. Ioannis I, Emmanouil V, Nadia TL, Minas L. A retrospective analysis of the characteristics, treatment and follow-up of 26 odontomas in Greek children. Journal of Oral Science. 2010;52(3):439-447.

6. Philipsen HP, Reichart PA, Praetorius F Mixed odontogenic tumours and odontomas. Considerations on interrelationship. Review of the literature and presentation of 134 new cases of odontomas. Oral Oncol. 1997;33:86-99.

7. Nelson BL, Thompson LD. Compound odontoma. Head Neck Pathol. 2010;4:290-291.

8. de Oliveira BH, Campos V, Marçal S. Compound odontoma-diagnosis and treatment: three case reports. Pediatr Dent. 2001;(23):151-157.

9. Amado-Cuesta S, Gargallo-Albiol J, Berini-Aytes L, Gay-Escoda C. Review of 61 cases of odontoma. Presentation of an erupted complex Odontoma. Med. Oral. 2003;8:366-373.

10. Urvashi S, Rahul S, Anubha G, Renu Y,Krishan G. Compound composite odontoma with unusual number of denticles-A rare entity. The Saud Dental Journal. 2010;22:145-149.

11. Pokharel S, Li Z. Teen gets 232 "teeth" removed in Mumbai. Retrieved July 25, 2014 from: http:// edition.cnn.com/2014/07/25/world/asia/indiaabnormal-teeth/

12. An S, An C, Choi K. Odontoma: a retrospective study of 73 cases. Imaging Sci Dent. 2012;42:7781. 